\title{
EMPODERAMIENTO Y CALIDAD DE ATENCIÓN EN SALUD ${ }^{1}$
}

\author{
(Empowerment and quality of health care)
}

\author{
Leda Berardi ${ }^{2}$ \\ (Universidad de Chile)
}

\begin{abstract}
"Un enfoque crítico en salud pública (...) se preocupa fundamentalmente de las prácticas sociales de poder y de cómo estas prácticas (políticas, económicas, culturales) funcionan para estratificar a los individuos en jerarquías y, de esta manera, estratificar sus riesgos, vulnerabilidad y acceso a los recursos de salud” (Green y Labonté 2008:10).
\end{abstract}

\begin{abstract}
The Chilean Ministry of Health has implemented policies to promote citizenship participation in and control over the health system. Changes in the behaviour of both health professionals and officers and users have had positive and negative effects on the relationship between them. As users have become more aware of their citizenship rights, they have become not only more assertive but have also caused some conflicts with the aforementioned. Taking into account all of these considerations and international evidence which shows a strong relationship between health system users empowerment and quality of health care, a series of interviews to health

1. Este trabajo corresponde a una primera fase del análisis crítico del discurso del personal del Servicio Salud Metropolitano Occidente (SSMO), Santiago de Chile.

2. Profesora de Inglés de la Universidad de Chile, Msc en Educación de la Universidad Central de Venezuela (UCV) donde también realizó estudios de Lingüística y Análisis del Discurso. Es académica de la Escuela de Salud pública de la Universidad de Chile, de la Universidad Academia de Humanismo Cristiano y de la Universidad ARCIS. Actualmente, es (C) a Dr. en Comunicación Lingüística y Mediación multilingüe, mención Análisis del Discurso. Universidad Pompeu Fabra, España.
\end{abstract}


professionals and officers were conducted in the Western Health Service of the Region Metropolitana and analyzed using Critical discourse analysis $(C D A)$ in order to determine their roles on users participation.

Key words: Empowerment, quality of Chilean public health care system, Appraisal theory, social determinants of health, $C D A$

\section{RESUMEN}

El Ministerio de salud de Chile ha implementado políticas para promover la participación y el control ciudadano en salud con el fin de mejorar la calidad de atención en salud. Los cambios experimentados, tanto por el personal que trabaja en salud como por los usuarios, han causado efectos positivos y negativos en la relación entre ambos. A medida que los usuarios han tomado mayor conciencia acerca de sus derechos ciudadanos, han llegado a ser no sólo más asertivos sino que también han causado algunos conflictos con el personal de salud. Tomando en cuenta esta situación y la evidencia internacional acerca del empoderamiento de los usuarios del sistema de salud para mejorar la calidad de la atención, se efectuaron una serie de entrevistas al personal del Servicio salud metropolitano occidente y se realizó un análisis crítico del discurso del estas para determinar el rol que desempeña dicho personal en el empoderamiento de los usuarios.

Palabras-clave: Empoderamiento, calidad de atención del sistema público de salud chileno,

Teoría de la valoración, determinantes sociales de la salud, análisis crítico del discurso.

\section{Introducción}

Cuando Izabel Magalhães me invitó a formar parte de la mesa temática "Transdisciplinaridad y análisis del discurso en América latina” desde el discurso político, no dudé en aceptar la propuesta por cuanto era una nueva oportunidad para (re) afirmar, una vez más, ${ }^{3}$ la necesidad de abordar de manera inter y transdisciplinaria los problemas u objetos de análisis mediante el análisis crítico del

3. Ver Berardi 2005 y 2009. 
discurso (ACD). De esta manera, las perspectivas o puntos de vista del/la analista del discurso se amplían y enriquecen, tanto a nivel teórico como metodológico.

Como consecuencia de lo anterior, el equipo de investigación dispondrá de herramientas y visiones más holísticas para proponer cambios, meta última del ACD. El objeto o problema que será investigado debiera surgir de necesidades sociales detectadas e (idealmente) respaldadas por los organismos, instituciones o comunidades que usarán los resultados del análisis para tomar decisiones respecto de una determinada situación.

El problema que se plantea en esta investigación “¿Contribuye el empoderamiento a la calidad de atención en salud?” debe necesariamente ser abordado de manera inter y transdisciplinaria por cuanto se requieren conocimientos provenientes no sólo del campo del ACD sino también del ámbito de la salud, de las políticas públicas y de las ciencias sociales con el fin de aportar antecedentes del contexto socio-económico y político para su comprensión e interpretación. Para ello, seguiré a van Leeuwen (2005:8), quien señala que el modelo integracionista se centra en problemas, por lo que "ninguna disciplina puede resolver por sí sola un problema determinado”.

Respecto del concepto de transdisciplinaridad, adopto la definición de Nicolescu (1998) quien la concibe como un proceso mediante el cual se trascienden los límites de las disciplinas individuales para tratar problemas desde perspectivas múltiples, con el fin de generar conocimiento emergente. Por su parte, Taborda et al. (2005) entienden la interdisciplinaridad como el análisis de problemas o conjuntos de problemas desde distintas miradas científicas con el fin de comprender las múltiples y distintas dimensiones de la realidad social.

Para responder la pregunta planteada en esta investigación, se analizarán las opiniones del personal que trabaja en el Servicio de salud metropolitano occidente (SSMO), es decir, el discurso que emana de las directrices gubernamentales, específicamente de los funcionarios y los profesionales de la salud dependientes del Ministerio de salud (MINSAL). 


\section{El Problema}

De acuerdo a información reportada por la Unidad de participación social de la Subsecretaría de Redes Asistenciales del Ministerio de salud (MINSAL) en el año 2007, el porcentaje de usuarios del sistema de salud que reclaman por el trato recibido asciende a $29 \%$. El tipo de reclamos que le sigue se relaciona con el tiempo de espera: $22 \%$.

A su vez, el informe ejecutivo basado en la "Encuesta nacional de derechos ciudadanos" y presentado a la Comisión Asesora Presidencial para la Protección de los Derechos de las personas por la Consultora Gestra en julio de $2008^{4}$ señala que los Consultorios y los Hospitales públicos reciben evaluaciones bajas (inferior a $60 \%$ de notas 6 y 7 ). En dichas dependencias el porcentaje de necesidad de reclamo es de 45,5 $\%$ y las principales razones esgrimidas para no concretar el reclamo están relacionadas con la percepción de "que no se consigue mucho en reclamar". Como consecuencia, sólo 1 de cada 3 personas reclama.

En los hospitales públicos, donde se aduce mal trato ("poca amabilidad", "prepotencia") y demora en la atención, la tasa de respuesta a los reclamos alcanza el $35.9 \%$. También se observa discriminación relacionada con "no ser nadie importante", "ser pobre", "ser joven”, "no tener educación” y "apariencia física”.

Respecto de la percepción de la calidad de la atención de salud que tienen las personas pobres de Santiago, Yánez (2007) -a partir de los resultados de una encuesta aplicada en las 22 comunas más pobres de la capital- señala que el $63,8 \%$ de los entrevistados estima que es "mala" o "muy mala". El 37,5\% reclama que el personal administrativo lo trató mal; el 32,8 responde que no le solucionaron el problema frente a un reclamo y el $18,8 \%$ que el médico lo trató o atendió mal. En relación a la discriminación, el 48,5\% cree que los trabajadores de la salud discriminan.

4. Esta encuesta consideró 1.803 entrevistas en todas las regiones del país y uno de los resultados fue que un $43 \%$ desconoce que puede exigir derechos al atenderse en los servicios públicos. 
A partir de estos datos, he considerado importante analizar las percepciones y opiniones presentes en el discurso del personal que trabaja en el SSMO de la Región metropolitana (RM) del país, con el fin de detectar el rol de dicho personal en el empoderamiento ${ }^{5}$ de los usuarios y si este influye en la calidad de la atención prestada. La importancia de conocer la incidencia del empoderamiento de los usuarios en la calidad de la atención recibida se basa en los resultados de múltiples publicaciones en las que se reporta que el empoderamiento de la población contribuye a mejorar sustancialmente la calidad de la atención en salud (Wallerstein 2002, 2006; Sen G. 1997; Rifkin et al. 1988; Narayan 2002,2005, entre otros).

Wallerstein (2006:1) señala que "las estrategias de empoderamiento del paciente y la familia han incrementado las habilidades de los pacientes para manejar su enfermedad, (...) usar los servicios de salud de manera más efectiva y también han incrementado las habilidades y eficacia del equipo de salud" (énfasis mío).

La autora define el empoderamiento como un "proceso de acción social que promueve la participación de la gente, organizaciones y comunidades hacia las metas de incrementar el control individual y de la comunidad, la eficacia política, (...) la calidad de la vida comunitaria, (la) justicia social, (y) participar en la vida democrática de las comunidades para el cambio social" (Wallerstein 1992, en De los Reyes 2007:7).

Lo que se plantea en el objetivo estratégico de la Unidad de participación social de la Subsecretaría de redes asistenciales del MINSAL coincide con lo expresado por Wallerstain $(1992,1996)$ acerca del empoderamiento. En dicho objetivo se especifica que el desarrollo de una política de participación debe "estimular la participación social y el control ciudadano en salud (con el fin de) generar respuestas adecuadas de la red de servicios de salud a las necesidades de la población, en un

5. El concepto se origina en Estados Unidos en los años sesenta durante la lucha de los derechos civiles de los afroamericanos y, a mediados de los setenta, comienza a aplicarse en los movimientos feministas, y por Freire en la lucha por los oprimidos de América latina (Denman et al. 1999) 
marco de equidad, calidad y respeto por los derechos y deberes de las personas" (énfasis mío).

A su vez, el objetivo sanitario del país "proveer servicios acordes a las expectativas de la población” también incluye aspectos relacionados con los derechos de los pacientes, tales como respeto a la dignidad y autonomía, y calidad de la atención (El Vigía 2002).

Acorde con lo expresado anteriormente, este trabajo también tuvo como propósito determinar la ideología subyacente en los discursos con el fin de develar los valores y creencias que sustentan "un orden político determinado, legitimizan jerarquías existentes y relaciones de poder, y preservan identidades de grupo" (Chiapello y Fairclough 2002:187).

\section{Contexto socio-económico}

En el seminario de psicología de la Universidad Santo Tomás, realizado en octubre de 2009, Benito Baranda, Director social nacional del Hogar de Cristo, señaló que el Informe Hábitat de la Organización de Naciones Unidas (ONU) determinó que Santiago es una capital fragmentada y segregada, donde un $7 \%^{6}$ de la población vive en asentamientos precarios. Agregó que "en Chile existe una desintegración social por la falta de oportunidades y deficientes políticas públicas. Las personas en riesgo social son excluidas y discriminadas, y 4 de cada 10 niños tiene una formación deficiente, lo que los priva, desde la infancia, de tener libertad y capacidad de tomar sus propias decisiones" (Universia 2009:1). Criticó la ausencia de participación ciudadana ${ }^{7}$, como en el caso de la erradicación de campamentos ${ }^{8}$, la segregación

6. Correspondiente a 1.120 .000 personas.

7. Respecto de la participación ciudadana en Chile, consultar Berardi 2009.

8. "Un Techo para Chile" define los campamentos como 8 o más familias agrupadas o contiguas, en una situación irregular de terreno y que carecen de al menos un servicio básico (luz, agua potable, alcantarillado). Según un informe de la Universidad Católica realizado en el 2009 por Unicef y la organización "Un techo para Chile", 20.000 familias y 50.000 niños y adolescentes viven en malas condiciones en 50 campamentos en Chile y el 13,3\% de ellos ha abandonado sus estudios 
social a través de la educación, y la acentuación de los beneficios materiales por sobre los procesos socio-comunitarios.

Respecto de la salud, una publicación de la Universidad de Los Lagos reporta que en el sistema público de salud se observa un déficit y una distribución desigual de recursos humanos, lo que incide en los tiempos de espera de los usuarios y en la calidad de la atención recibida en los centros de atención primario y secundario. El déficit en acceso, cobertura y calidad de prestaciones, tanto en el ámbito público como privado, no satisface adecuadamente la demanda existente. En el sector privado, los planes y programas de salud a los que pueden optar las personas que cotizan en Isapres ${ }^{9}$ dependen del ingreso del afiliado. Aun cuando la calidad de atención suele ser mayor, este sistema presenta déficit de protección, transparencia y altos costos para el cotizante.

Las siguientes cifras y datos entregados por Pizarro (2004) dan cuenta de la desigualdad existente en el ámbito de la salud: i) la mortalidad infantil en Puerto Saavedra ${ }^{10}$ es 14 veces mas alta que en Vitacura ${ }^{11}$ y en Independencia ${ }^{12}$ es siete veces mas alta que en Vitacura; ii) las expectativas de vida en zonas pobres son entre 10 a 20 años inferiores en relación a las ricas; iii) dos tercios de las horas médicas se dedican al sistema privado que cubre menos de un tercio de la población; iv) los recursos que FONASA ${ }^{13}$ destina por persona para atender a sus cotizantes y a los indigentes son inferiores a los de las ISAPRES; v) los costos de administración de estas últimas son muy superiores a los de FONASA; vi) el servicio público debe atender muchas veces a los usuarios de las ISAPRES, lo que debilita el quehacer público con los más desamparados; vii) las ISAPRES no atienden a las personas que no les son rentables:

9. Sistema privado de salud

10. Más del $75 \%$ de su población es pobre

11. Vitacura es un sector de la capital con más hogares $\mathrm{ABC}$, es decir, con mayores recursos económicos (Diario La Segunda, 4 mayo, 2004)

12. En la comuna de Independencia, $34,6 \%$ de la población pertenece al estrato social “D” o pobre, con un ingreso que fluctúa entre US\$ 475 y US\$ 860

13. Fondo nacional de salud (sistema público de salud) 
ancianos, embarazadas y personas con enfermedades catastróficas. A su vez, el senador Mariano Ruiz Esquide, integrante de la Comisión de salud del Senado, agrega que el sistema público atiende a aproximadamente el $70 \%$ de la población con la mitad del financiamiento per cápita y ese $70 \%$ está compuesto mayoritariamente por pobres, ancianos y niños, que presentan alta morbilidad. El sector privado, con el doble del financiamiento per cápita, atiende un universo fundamentalmente joven, "de los más altos ingresos del país, y de morbilidad menor, con el agravante de no asumir la prevención para sus propios afiliados, que son de costo del sector público estatal”. (Fundación Terram 2004).

\section{Antecedentes teóricos y metodológicos}

Las fuentes teórico-metodológicas utilizadas para realizar esta investigación derivan de Green y Labonté (2008), Martin (2000), Wallerstein (2002, 2006), Batliwala (1999), Stromquist (1999) van Leeuwen ( 2005), Chiapello y Fairclough (2002), Kaplan (2007), White (2003) y Bakhtin (1981). El análisis de los discursos se abordará desde la Teoría de la valoración, definida como "un enfoque particular para explorar, describir y explicar cómo el lenguaje se usa para evaluar, adoptar posturas (intersubjetivas) y construir posiciones y relaciones interpersonales. (...) Explora cómo las actitudes, juicios y respuestas emocionales se presentan explícita o (implícitamente) en los textos" (Martin 2000:1).

Los recursos evaluativos provenientes de dicha teoría se dividen en tres dominios semánticos: la actitud, el compromiso y la gradación. Los enunciados actitudinales expresan una evaluación positiva o negativa de personas y procesos e incluyen los subsistemas de afecto, juicio y apreciación.

Para los fines de este trabajo, sólo se recurrirá a los subsistemas de juicio y apreciación, y al dominio del compromiso. El juicio es "la evaluación del comportamiento humano con respecto a normas 
sociales institucionalizadas" (White 2003:3). Un juicio se puede expresar de manera directa, mediante la utilización de adverbios, atributos y epítetos, sustantivos y verbos. También puede ser indirecto, utilizando indicadores (tokens) de juicio que pueden activar una evaluación actitudinal de parte del que escucha o lee, según su postura ideológica. ${ }^{14}$ La apreciación es la evaluación de objetos naturales y manufacturados, procesos, textos y constructos como planes y políticas, (...) en función de sistemas de valor social (Martin 2000). Por su parte, el compromiso está constituido por los recursos mediante los cuales el emisor "expresa, negocia y naturaliza ${ }^{15}$ posturas particularmente intersubjetivas (con los receptores) que son, en última instancia, ideológicas" (White 2003:8). De aquí la noción de "negociación heteroglósica"16 entendida como una "perspectiva que enfatiza el rol del lenguaje para posicionar a los emisores de textos en la heterogenidad de las posturas sociales y visiones de mundo que operan en una cultura determinada" (White 2003:16). De acuerdo a la heteroglosia, los enunciados reconocen la existencia de otras voces y posturas alternativas. Desde esta perspectiva, la modalidad puede utilizarse para indicar que el enunciado puede ser negociado con personas que ostentan posturas diferentes.

Acorde con lo anterior, también se mostrarán los recursos lingüísticos y las estrategias discursivas utilizadas por los entrevistados para asumir roles de funcionarios gubernamentales y/o de ciudadanos, y para transmitir posturas ideológicas.

Respecto del concepto de empoderamiento, seguiré a Green y Labonté (2008), Stromquist (1999), Blatiwala (1999) y Wallerstain (2006). Las propuestas de Green y Labonté (2008) me permitieron abordar dicho concepto desde una perspectiva crítica que pone en

14. Aun cuando el enunciado le disparó a quemarropa no contiene en sí mismo elementos evaluativos, activa una respuesta interpersonal de rechazo (Kaplán 2007).

15. Los recursos retóricos intentan persuadir de manera indirecta, haciendo parecer naturales las creencias y supuestos.

16. La noción de heteroglosia deriva de Bakhtin (1981) 
tela de juicio algunos de los planteamientos incluidos en el objetivo estratégico de la Unidad de participación social de la Subsecretaría de redes asistenciales del MINSAL, reseñado anteriormente.

El Ministerio delega en el ciudadano la responsabilidad de la calidad de la atención recibida, al situar al ciudadano como un agente de control del sistema de salud para que este pueda proporcionarle una atención de calidad. Al respecto, Green y Labonté (op. cit.:6) señalan que "las políticas públicas en salud (...) centran la responsabilidad de resolver problemas de desigualdad precisamente en las personas más vulnerables (...) y con ínfimas posibilidades para poder cambiar los determinantes estructurales de la salud”. Agregan que la apelación a la participación social "puede correr el riesgo de ignorar (...) las desigualdades sociales de las diferentes comunidades e identidades sociales".

En el resumen analítico "Alcanzar la equidad sanitaria actuando sobre los determinantes sociales de la salud", del informe final de la Comisión sobre Determinantes Sociales de la Salud de la Organización Mundial de la Salud (2008:5) se reporta que "los determinantes estructurales y las condiciones de vida en su conjunto constituyen los determinantes sociales de la salud, que son la causa de la mayor parte de las desigualdades sanitarias entre los países y dentro de cada país" (énfasis mío). Por ende, los determinantes sociales en salud constituyen una variable fundamental que debe ser tomada en cuenta para proponer acciones en el ámbito de la calidad y equidad en salud.

La misma Comisión agrega que "la mala salud de los pobres, el gradiente social de salud dentro de los países y las grandes desigualdades sanitarias entre los países están provocadas por una distribución desigual, a nivel mundial y nacional, del poder, los ingresos, los bienes y los servicios, y por las consiguientes injusticias que afectan a las condiciones de vida de la población de forma inmediata y visible (acceso a atención sanitaria, escolarización, educación, condiciones de trabajo y tiempo libre, vivienda) y a la posibilidad de tener una vida próspera. Esa distribución desigual de experiencias perjudiciales para la salud (...) es (...) el resultado de una nefasta combinación 
de políticas y programas sociales deficientes, arreglos económicos injustos y una mala gestión política” (énfasis mío).

De aquí la importancia de que las políticas públicas en salud deban priorizar los determinantes sociales de la salud antes que la responsabilidad individual de los ciudadanos, tanto respecto de su propia salud como de la calidad de la atención recibida.

Pizarro (op. cit.), por su parte, especifica que la pésima distribución del ingreso ostentada por $\mathrm{Chile}^{17}$ se debe a la concentración patrimonial en 16 grupos económicos en cuyas manos se encuentra el $80 \%$ del producto nacional. ${ }^{18}$ No obstante lo anterior, la Organización para la Cooperación y el Desarrollo Económico (OCDE), aun cuando reconoce que la clase media chilena se caracteriza por tener una de las peores distribuciones del ingreso en latinoamérica (Claude, 2007) ${ }^{19}$, decidió incluir a Chile al grupo de las naciones más desarrolladas del mundo, invitación que fue confirmada por el Gobierno en enero del 2010.

Algunas de las recomendaciones de la OCDE para Chile en lo referente al empleo implicarán un mayor compromiso de Chile con el modelo económico neo-liberal, lo que incrementaría aún más las desigualdades en la distribución del ingreso ${ }^{20}$. Ampliar el seguro de desempleo, disminuir los costos de despidos, pagar menos que el sueldo mínimo $^{21}$ a las personas menores de 25 años y tener mayor rigidez del sistema de contratación y despidos implicarán más desigualdad e inequidad respecto de los determinantes sociales de la salud.

17. Chile ocupa el lugar 11 entre los 12 países con peores distribuciones del ingreso en el mundo, entre Paraguay y Etiopía (Berardi 2009)

18. Respecto de los sentimientos de la ciudadanía derivados de esta situación, consultar Berardi 2009

19. La OCDE está integrada por los 30 países más desarrollados del mundo.

20. Sin embargo, Andrés Velasco, Ministro de economía del gobierno de la Presidenta Bachelet, señaló que el ingreso de Chile a la OCDE "es un reconocimiento para Chile (por cuanto) es una asociación de países democráticos, con las instituciones más fuertes, los estados más transparentes y los mejores instrumentos para luchar contra la pobreza y la desigualdad” (énfasis mío). (Radio Bio Bio, 15 diciembre 2009).

21. En junio del 2009 aumentó a U\$ 317. 
En lo concerniente a estrategias de empoderamiento, Wallerstain (2006) especifica que estas deben considerar la acción comunitaria a través de su incorporación y participación en las fases de planificación, implementación y evaluación de las políticas de salud pública. De esta manera, las necesidades concernientes a la salud son determinadas por la comunidad, privilegiándose, así, una participación "desde abajo" (bottom-up), en contraposición a la sóla oferta de servicios.

\section{El Metodo}

Las categorías de análisis utilizadas para cumplir con el objetivo de esta investigación son:

1. Empoderamiento

Entendido como el proceso mediante el cual la ciudadanía aprehende conocimientos y estrategias que le permita participar en las etapas de planificación, operacionalización y evaluación de las políticas públicas en salud (Stromquist 1999).

2. Determinantes sociales de la salud

El denominado enfoque integral en salud (Franco 2001) considera a la salud como un proceso construido socialmente en el que no sólo se considera a la salud relacionada con los estilos de vida sino que incluye los determinantes sociales de la salud, definidos como las condiciones de vida que afectan a la salud.

Siguiendo a Franco (2001:1), existe una estrecha relación entre las enfermedades y los problemas sociales. Señala que varios autores reconocen que "el cuerpo siente y expresa problemas sociales”, (y que la) percepción de situaciones adversas resultan nocivas para la salud de las personas, llamándose a esto 'lesiones de vida'”. Agrega que "la evidencia demuestra que un problema de salud no puede 
solucionarse sin resolver las diferentes circunstancias que rodean a las personas"

3. Calidad de atención en salud

El Minsal, en el resumen ejecutivo de los Objetivos sanitarios para la década 2000-2010, define la calidad de la atención como "la aplicación de la técnica y tecnología médica en una forma que maximice sus beneficios para la salud, sin aumentar en forma proporcional sus riesgos”. Especifica que "diversos estudios demuestran (...) que los chilenos están disconformes con los servicios que reciben y que se sienten desprotegidos frente a eventuales necesidades de salud. No obstante estas evidencias, se adolece de un sistema de información que permita establecer la situación basal a cabalidad y monitorear su evolución”. (El Vigía: 11)

En contraste con lo anterior, define las expectativas de la población como los componentes no médicos de la atención e incluye aspectos relacionados con los derechos $\mathrm{d} e$ los pacientes, como el respeto a la dignidad, la autonomía y la confidencialidad, así como la orientación al usuario respecto de la pronta atención, las comodidades básicas, la posibilidad de contacto con familiares cercanos durante el proceso de atención y la capacidad de elección.

Para los efectos de esta investigación, la calidad de la atención en salud será entendida como respeto por los derechos de los usuarios tanto en su relación con los profesionales de la salud como con las instancias administrativas de atención al usuario.

\section{El Corpus}

El corpus de esta parte de la investigación está constituido por el discurso de un funcionario (FOR) perteneciente a la Oficina de información y reclamos en salud (OIRS) y el discurso de un 
funcionario del Departamento de Recursos humanos (FRH) del Servicio Salud Metropolitano Occidente (SSMO).

Se seleccionó el personal de este Servicio por las características socioeconómicas y de salud de los usuarios. Dicha población "presenta una situación desmedrada en relación a otros servicios de la RM ya que un tercio de sus habitantes vive en hogares calificados como "pobres". (...) A nivel urbano, las comunas más pobres son Cerro Navia y Renca y, conjuntamente con la comuna de Lo Prado, presentan las mayores tasas de desocupación. (...). Estas comunas muestran la mayor concentración de problemas de salud. (...) El Servicio (también) presenta porcentajes de analfabetismo superiores al promedio de la RM y al país" (Presentación Servicio Salud Metropolitano Occidente 2008: 20, énfasis mío). Para recolectar la información se utilizaron Entrevistas en profundidad no estructuradas a los dos funcionarios anteriormente identificados.

\section{Análisis e interpretación de los datos}

El funcionario de la Oficina de Reclamos (FOR) señala que existe un $30 \%$ de reclamos a nivel nacional. El primer lugar lo ocupa el trato, seguido por el tiempo de espera, el conocimiento del diagnóstico, los procedimientos técnicos y el Régimen de Garantías en Salud (GES) que cubre 56 problemas de salud catastróficos, vigente desde el 1 de julio de 2006.

\section{Calidad de atención en salud}

Entrevistadora:

"Qué entienden por calidad de atención de salud?" FRH

(1) "Hacer las cosas bien a la primera, es una cultura, estilos de vida, estándares de calidad en salud, implica un trabajo de mejoramiento continuo; no debiera tener techo el estándar. (Es) un estado de trabajo continuo". 
El FRH responde desde su rol de funcionario realizando un juicio implícito o indirecto negativo de los profesionales de la salud y una apreciación negativa de las operacionalización de las políticas públicas en salud. "Hacer las cosas bien a la primera" evoca que se está enjuiciando negativamente lo que sucede actualmente ${ }^{22}$ respecto de la calidad de atención en salud.Con la utilización del modal "debiera" se evidencia una estrategia retórica heteroglósica con el fin de establecer una negociación heteroglósica, es decir, el hablante se abre a la posibilidad de negociar este punto de vista con 1@s interlocutor@s.

\section{FOR}

(2) "La gente evalúa mejor el trato que las competencias. Uno busca alguien en quien confiar".

El funcionario de la OIRS emite un juicio implícito negativo del comportamiento de la gente y un juicio implícito negativo respecto de las competencias del personal de salud. Por otra parte, al utilizar el pronombre "uno" asume el rol de ciudadano, compartiendo, así, la evaluación de la ciudadanía respecto del trato.

(3) "(Deben implementarse) programas de capacitación para mejor atención del usuario".

Se observa una apreciación negativa de la puesta en práctica de las políticas públicas en salud (para mejor atención del usuario). A su vez, no sólo no se incluye - desde su rol de funcionario gubernamental - entre los agentes o actores que deben implementar los programas aludidos, sino que, además, dichos actores no se identifican.

ENTREVISTADORA: Los usuarios del sistema público de salud se quejan de que los profesionales de la salud los maltratan ¿Qué opinan Uds. al respecto?

22. Las entrevistas se realizaron en el año 2009. 


\section{FOR}

(4) "El maltrato es subjetivo, puede haber un problema de empatía. (...) Hay cosas que son más objetivadas. (...) Maltrato implica algo violento".

Desde su rol de funcionario de la OIRS, mediante la utilización del atributo violento emite un juicio directo negativo de los usuarios. Aun cuando negocia el significado utilizando el modal "puede" - produciendo el mismo efecto respecto del modal debiera consignado anteriormente en (1) - al considerar el maltrato como un asunto de orden tangible (Hay cosas que son más objetivadas), legitima el rol de los profesionales de la salud, por cuanto no considera el maltrato verbal.

\section{FRH}

(5) "Una explicación de lo que yo entiendo por maltrato (es que) ocurre por formación, personalidad, circunstancias, oportunidad de elegir (donde trabajar)”

El entrevistado se posiciona en el rol de sicólogo ${ }^{23}$ o ciudadano (yo entiendo) en lugar del de funcionario de recursos humanos. La utilización del verbo entiendo explicita lo que Bakhtin (1981) denomina la voz subjetiva del autor, lo que implica que es sólo una voz entres otras posibles. A su vez, White 2003:272) lo considera un recurso de intra-vocalización de la heteroglosia denominado proclamación/pronunciamiento. De esta manera, el emisor negocia heteroglósicamente con los interlocutores para asumir la responsabilidad de lo dicho, con el propósito de que el enunciado permita la interacción con la diversidad heteroglósica.

Por otra parte, transfiere la responsabilidad del maltrato a la institución que formó al profesional de la salud (ocurre por formación) y al modo de funcionamiento del sistema público de salud (oportunidad de elegir). Emite, así, una apreciación negativa respecto

23. En Chile, los profesionales relacionados con recursos humanos suelen ser sicólogos. 
de las políticas en salud y un juicio indirecto negativo acerca de los profesionales (el maltrato ocurre por personalidad)

(6) "En el (sistema) privado se podría alegar maltrato porque no le cambió el canal de TV"

El funcionario verbaliza una apreciación positiva indirecta de las políticas en salud por contraste implícito. Además, al igual que en (1) y (4), el modal podría permite una negociación heteroglósica con los interlocutores.

(7) "El consentimiento informado se dejó como un ejercicio no escrito y era la palabra del médico contra la palabra del usuario".

Aun cuando no se critica explícitamente el accionar gubernamental respecto del consentimiento informado, el enunciado se dejó como un ejercicio no escrito evoca una situación de incompetencia respecto de las políticas en salud, y, por ende una apreciación negativa de estas. A la vez, mediante un juicio directo (era la palabra del médico contra la palabra del usuario) evalúa negativamente a los médicos.

\section{Empoderamiento}

ENTREVISTADORA: El Minsal señala que se debe promover la participación social en salud para que los servicios de salud funcionen con equidad, calidad y se respeten los derechos y deberes de los usuarios. ¿Cómo se ha implementado esta política?

\section{FOR}

(8) "La participación en salud se abre con el Hospital amigo $(\mathrm{HA})^{24}$. Cuando se abre, ¿dónde van a comer, dormir (los

24. En el año 2007, el subsecretario de Redes Asistenciales, Ricardo Fábrega, señaló que todos los recintos médicos debían implementar el HA, lo que implicaría gastos en infraestructura y mayor disposición a atender público por parte del personal. La idea del Ministerio de Salud consistió en dar un espacio a la familia en la recuperación de los enfermos (Radio Cooperativa 2007). 
familiares)? Se privilegia a los niños y adultos mayores. Se genera una presión en los funcionarios, técnicos, enfermeras, por los parientes".

El hablante emite una apreciación negativa de la operacionalización de las políticas en salud respecto del HA. También enjuicia negativamente a los parientes de los usuarios.

\section{FRH}

(9) “(Hubo) resistencias iniciales al HA (...) Tiene que haber ciertas condiciones. El técnico paramédico está acompañado por los parientes que están supervigilando. Otros (profesionales de la salud) lo interpretan como colaboración del usuario"

Se observa una apreciación negativa de la puesta en práctica de las políticas públicas en salud respecto del HA (hubo resistencias iniciales; tiene que haber ciertas condiciones). También expresa un juicio positivo acerca de la conducta de otros profesionales de la salud (otros lo interpretan como colaboración del usuario), y un juicio indirecto negativo de los parientes (los parientes que están supervigilando).

FOR

(10)' "En el HA los familiares tienen derecho a estar con los parientes".

El funcionario evalúa positivamente las políticas respecto del HA, emitiendo una apreciación positiva (los familiares tienen derecho).

(11) "Debe haber gestión participativa en salud, es un paso cultural que debe dar Chile”.

(12) "El empoderamiento del usuario es un camino que debe hacerse en conjunto (con los profesionales y administrativos de la salud)" 
(13) "El usuario participa en cuentas públicas"

En (11) y (12), el FOR realiza una apreciación negativa de las políticas públicas en salud relacionadas con la ausencia de participación ciudadana en dicho ámbito. Aun cuando en (13) se observa una apreciación positiva respecto de las políticas de participación, la ciudadanía en general y los usuarios en particular rara vez buscan en Internet asuntos relacionados con cuentas públicas. Legitima, así, el sistema de creencias y valores que subyacen a la política de gobierno.

(14) “(Al) poner caderas a pacientes de 80 años, están más garantizados los mayores que los de 40 o 50 años. En salud se debe discutir con la comunidad y no ser tan autócratas".

El funcionario emite una apreciación negativa de las políticas en salud concernientes al GES (están mas garantizados los mayores que los de 40 o 50 años). También se posiciona en el rol de ciudadano para expresar un juicio directo ${ }^{25}$ negativo acerca del accionar del Minsal a través del atributo "autócrata" (en salud se debe discutir con la comunidad y no ser tan autócratas)

\section{FRH}

(15) "El usuario que sabe que tiene derechos por publicidad, GES, discurso ciudadano, exige sus derechos y hay complicaciones (...), lo hace con cierta soberbia”.

Se aprecia una evaluación negativa del comportamiento de los usuarios mediante la utilización de un sustantivo (complicaciones) y un adverbio (soberbia) que implican un juicio directo. Por otra parte, el uso de la generalización "el usuario exige sus derechos y hay complicaciones (...) lo hace con cierta soberbia" pone en evidencia una ideología que legitima tanto la

25. Debido a la ausencia de sujetos explícitos, también se podría interpretar como una apreciación negativa de las políticas en salud 
jerarquía existente entre los usuarios y el Minsal como su rol de funcionario gubernamental.

(16) "El Minsal generó una publicación: 'Hagamos un trato, tratémonos bien'. Esperamos un buen trato de vuelta"

Además de una apreciación positiva acerca del Minsal (el Minsal generó una publicación: 'Hagamos un trato, tratémonos bien'), el emisor utiliza una estrategia de inclusión del usuario (hagamos un trato, tratémonos bien) para que la responsabilidad atingente al maltrato sea compartida, dejando en un plano de igualdad los problemas derivados del sistema de salud. Por otra parte, mediante la afirmación "esperamos un buen trato de vuelta" responsabiliza a los usuarios del maltrato por cuanto no incluye al personal de salud. Se legitima, de esta manera, una ideología que naturaliza la jerarquía entre el Ministerio y los usuarios.

\section{FOR}

(17) "El personal reclama cuando hay riesgo de integridad física o sicológica. Los funcionarios querían una OIRS para ellos para reclamar contra los usuarios. Se les dijo que tenían gremios”.

Tanto la afirmación los funcionarios querían una OIRS para ellos para reclamar contra los usuarios como la respuesta del Minsal (se les dijo que tenían gremios) constituyen juicios indirectos negativos del comportamiento de los funcionarios que activan una evaluación actitudinal negativa acerca de la conducta de los funcionarios. Al mismo tiempo, se legitima la jerarquía y relaciones de poder del Minsal y se naturaliza el funcionamiento de los gremios.

\section{FRH}

(18) "(Es necesario) que la gente se empodere para que ejercite sus derechos. El empoderamiento no se aprende de 
un día para otro". (Debe haber) empoderamiento de temas, de funciones"

Asumiendo el rol de ciudadano, se observa tanto un juicio indirecto negativo respecto de la gente (es necesario que la gente se empodere), como una apreciación negativa de la operacionalización de las políticas en salud (el empoderamiento no se aprende de un día para otro; debe haber empoderamiento de temas, de funciones).

\section{Determinantes sociales de la salud}

ENTREVISTADORA: "Cómo se relaciona la calidad de vida con el comportamiento de los usuarios respecto de la salud?"

\section{FOR}

(19) "Los usuarios demandan salud integral sin responsabilizarse. La salud está fuera, no dentro de los establecimientos"

(20) "Hay pacientes crónicos que no siguen los tratamientos; que tienen problemas con los estilos de vida"

(21) "El sistema de salud se transforma en reservorio de problemas sociales, de cosas que son rotulados como de salud pero no son. En siquiatría la familia abandona a los pacientes. (Hay) niños con malformaciones que son abandonados en los servicios y se gastan recursos en cama, agua, enfermera, etc."

Desde su rol de funcionario gubernamental, además de una evaluación negativa de los usuarios por medio de un juicio directo (se gastan recursos en cama, agua, enfermera, etc.), este no considera los determinantes sociales que pudieran ocasionar estas conductas (niños con malformaciones que son abandonados en los servicios; en siquiatría la familia abandona a los pacientes). En (21) formula 
apreciaciones negativas acerca de la operacionalización del modelo económico por parte del gobierno.

Mediante estrategias de transferencia ${ }^{26}$, en los tres enunciados la responsabilidad de mantener una vida saludable recae en los usuarios sin considerar las condiciones de vida que los afectan (usuarios demandan salud integral sin responsabilizarse; pacientes crónicos que no siguen los tratamientos, que tienen problemas con los estilos de vida; la salud está fuera, no dentro de los establecimientos; reservorio de cosas que son rotulados como de salud pero no son; el sistema de salud se transforma en reservorio de problemas sociales).

\section{FRH}

(22) "El nivel de desarrollo de los países (está dado por) la calidad de vida (que está condicionada por la salud). Salud no puede resolver la calidad de vida"

Desde el rol de ciudadano, se observa una apreciación negativa del nivel de desarrollo del país y, como funcionario, respalda las funciones inherentes al sistema de salud. Al igual que el FOR en (21), emite una apreciación negativa del funcionamiento del modelo económico chileno (salud no puede resolver la calidad de vida).

(23) "Es menos costoso llevar un equipo médico al paciente. Se está implementando un modelo de atención en el que equipos multidisciplinarios van a la comunidad. Los consultorios se están transformando en CES$\mathrm{FAM}^{27}$ : grupos de profesionales que están a cargo de (un área determinada); el equipo sale a terreno, realiza visitas domiciliarias a pacientes crónicos, (consideran) el estilo de vida”.

26. Para el uso de estrategias de referencia en el discurso político, consultar Berardi 1999

27. Centros de salud familiar. 
Como funcionario gubernamental, emite una apreciación positiva de las políticas públicas en salud atingentes a los CESFAM.

FOR

(24) "En lo privado, el usuario es un cliente porque paga, sólo hay oficina de información. Te internan en un spa, entran sin dientes y salen con ellos"

Se observa una apreciación negativa del sistema de salud privado (sólo hay oficina de información) y una apreciación positiva de las OIRS por contraste implícito.

"Hay ciertos cuestionamientos que forman parte de las circunstancias en las que se atiende. En lo privado es diferente (...) no se da el problema del tiempo"

(26) "En Oftalmología recuperan la vista, pero en lista de espera según la oferta y la demanda”.

En (25) el funcionario de la OIRS emite una apreciación positiva de las políticas en salud pública por contraste implícito; en (26) realiza una apreciación positiva de las políticas en salud pública por contraste.

\section{Consideraciones finales}

El análisis crítico del discurso de los funcionarios muestra un predominio de juicios y apreciaciones negativas acerca de las políticas públicas en salud. En un total de 26 enunciados, se observan 13 juicios negativos y 1 positivo; 10 apreciaciones negativas y 5 positivas acerca de las políticas en salud.

Respecto de los roles asumidos, los funcionarios se posicionan en el rol de ciudadano cada vez que realizan un juicio negativo acerca de los profesionales de la salud y los funcionarios del Minsal, o una 
apreciación negativa atingente a las políticas públicas en salud y a la operacionalización del modelo económico.

En lo que concierne a ideología, en ambos funcionarios se observan recursos lingüísticos que legitiman las creencias y valores que subyacen a la política de gobierno en salud mediante la naturalización de (i) un sistema de participación del usuario en salud, prácticamente inexistente (13); (ii) la jerarquía en las relaciones de poder entre el Minsal, los funcionarios y los usuarios (15), (16) y (17); y (iii) los gremios como entidades que defienden los derechos laborales de sus integrantes (17).

A su vez, tanto las estrategias de transferencia (los usuarios demandan salud integral sin responsabilizarse) como las de generalización (en siquiatría la familia abandona a los pacientes; los usuarios demandan salud integral sin responsabilizarse), justificación (en lo privado el usuario es un cliente porque paga; te internan en un spa, entran sin dientes y salen con ellos; hay ciertos cuestionamientos que forman parte de las circunstancias en las que se atiende), contraste (sólo hay oficina de información; en lo privado es diferente, no se da el problema del tiempo,) y exclusión (esperamos un buen trato de vuelta) se utilizan para transmitir el sistema de creencias y valores que sustentan el orden político imperante en la sociedad chilena. Los verbos modales en (1) y (6) y el verbo "entiendo" en (5) son utilizados por los dos funcionarios para negociar heteroglósicamente posturas personales e ideológicas con los interlocutores.

En relación al empoderamiento, los funcionarios lo vinculan a la participación del usuario en salud y señalan que su implementación ha causado problemas, especialmente a nivel de usuario. Especifican que esto se debe a la ausencia de gestión participativa y abogan por una política de empoderamiento que incluya tanto al personal de salud como a los usuarios. Respecto de la ausencia de participación social, Lechner (2002) señala que esta se deriva de la falta de cohesión social. Debido a la exclusión del bienestar económico, de las redes sociales y de una comunidad de sentidos, los ciudadanos no se perciben como parte de un sujeto colectivo que les permita internalizar un sentido común. 
Green y Labonté (2008:8) agregan que a los ciudadanos se les impone la participación comunitaria y el empoderamiento con el fin de que no sólo desarrollen una conducta de auto cuidado "orientada hacia su propia salud en lugar de (la salud) de la red social, (sino que también) asuman responsabilidades (...) hacia el cuerpo político”. Mientras no se implemente un proceso que cambie la distribución del poder ${ }^{28}$, "tanto en las relaciones interpersonales como en las instituciones de la sociedad” (Stromquist (1999:192), el empoderamiento seguirá siendo una meta inalcanzable y no podrá, por ende, contribuir a una mejor calidad de atención en salud.

Recebido em fevereiro de 2010 Aprovado em abril de 2010 leda.berardi@gmail.com

\section{Referncias bibliográficas}

BAKHTIN, M. The Dialogic Imagination: Four essays (Trads. C. Emerson y M. Holquist). Austin: University of Texas Press. 1981

BERARDI L. Democracia y salud en Chile. En Ferreira de Melo, I. (Ed.) Análise Crítica do Discurso: reflexões teóricas e aplicadas. En prensa. 2010 BERARDI L. Participación ciudadana y derechos sociales en Chile. En Bentivoglio, P., Erlich, F., y Shiro M. (Eds.) Haciendo discurso. Homenaje a Adriana Bolivvar. Caracas: Universidad Central de Venezuela, 2009

BERARDI L. Análisis del Discurso: Desafíos y propuestas. En Revista latinoamericana de estudios del discurso Vol. 5(1). 2005

28. El poder implica el control sobre los bienes materiales (trabajo y dinero), los recursos intelectuales (conocimiento, información e ideas) y la ideología. "El control sobre la ideología significa habilidad para generar, propagar, sostener e institucionalizar conjuntos específicos de creencias, valores, actitudes, y comportamientos, determinando la forma en que las personas perciben y funcionan en un entorno socioeconómico y político dado” (Blatiwala (1999:195). 
BERARDI, L. Globalization and poverty in Chile. Discourse \& Society 12, 1.47-58. 2001.

BLATIWALA, S. El significado del empoderamiento de las mujeres: nuevos conceptos desde la acción. En Denman c., Araneda, P., y Cornejo, E. Región y sociedad Vol. XI (18): 195, 1999.

CLAUDE, M. 2007. La inconclusa utopía de la democracia.

Disponible en www.marcelclaude.cl. (Consulta: 10 de enero 2007).

CHIAPELLO, E.; Fairclough, N. Understanding the new management ideology. A transdisciplinary contribution from critical discourse analysis and new sociology of capitalism. Discourse and Society, 13 (2): 185-208, 2002.

Comisión sobre Determinantes Sociales de la Salud de la Organización Mundial de la Salud. Alcanzar la equidad sanitaria actuando sobre los determinantes sociales de la salud, 2008. Disponible en whqlibdoc.who. int/hq/2008/WHO_IER_CSDH_08.1_spa.pdf. (Consulta: 12 de abril 2009).

Consultora Gestra. Encuesta nacional de Derechos Ciudadanos. 2008. Disponible en www.comisiondefensoraciudadana.cl (Consulta 5 mayo 2009).

De los Reyes. M.C. "Resistencia y empoderamiento de la sociedad civil ante el abuso sexual infantil en un contexto institucional". II Congreso nacional de Trabajo social y Encuentro latinoamericano de docentes, profesionales y estudiantes de Trabajo social. Mar del Plata, 2007.

DENMAN, C.; Aranda, P.; Cornejo, E. Reseña de Poder y empoderamiento de las mujeres de Magdalena León (comp.). Región y sociedad Vol. XI (18). México: El Colegio de Sonora, pp. 189-197

El Vigía. Boletín de vigilancia en Salud pública de Chile. Resumen ejecutivo. Objetivos sanitarios para la década 2000-2010. Vol. 5 No 15. 2002. Disponible en http://epi.minsal.cl/epidemiología (Consulta: 9 de abril 2009).

FRANCO, S. Análisis de la pertinencia social de la investigación en salud reproductiva período 1999-2001. Disponible en www.rec.uba.ar/Documentos/.../doc2InformeSFranco.doc (Consulta: 17 agosto 2009).

Fundación Terram 2004. Reforma a la salud ¿El ocaso de la solidaridad? Análisis de coyuntura social (ACDS), 16. Disponible en www.terram.cl (Consulta: 10 de marzo 2008). 
GREEN, J. y Labonté, R. Introduction: from critique to engagement. Why critical public health matters. En Green J. y Labonté, R (Ed.). Critical perspectives in Public health. NY: Routledge, 2008, p. 1-11.

KAPLÁN, N. La teoría de la valoración: un desarrollo de los estudios sobre la evaluación en el lenguaje. En Bolívar A. (Comp.) Análisis del discurso. Por qué y para qué. Caracas: Los Libros de El Nacional, 2007, p. 65-86

LECHNER, L. Los desafíos politicos del cambio cultural. Programa de las Naciones Unidas para el Desarrollo (PNUD), 2002. Disponible en www. desarrollohumano.cl (Consulta: 12 de marzo de 2007).

MARTIN, J. R. Beyond exchange: APPRAISAL systems in English. En Hunston S. y G. Thompson (eds.) Evaluation in text: Authorial stance and the construction of discourse. Oxford: Oxford University Press, 2000, p. 142175.

NARAYAN, D. (2005). Measuring Empowerment: Cross Disciplinary Perspectives.

Washington DC: Banco Mundial, 2005.

NARAYAN, D. (2002). Empoderamiento y reducción de la pobreza. Libro de consulta.

Coedición del Banco Mundial y Alfaomega Grupo Editor, Colombia. 2002

NICOLESCU B. La transdisciplinariedad, una nueva visión del mundo. Manifiesto. Paris: Ediciones Du Rocher. 1998.

Organización Mundial de la Salud. Informe final de la Comisión sobre Determinantes Sociales de la Salud. 2008

PIZARRO R. Desigualdad: el verdadero riesgo país. Centro de estudios sociales Avance

Ponencia presentada al Encuentro de economistas socialistas, 29-30 de mayo, 2004. Disponible en www.centroavance.cl (Consulta: 5 de mayo 2009)

RIFKIN, S. y Bichmann, W. (1988) 'On Measuring Participation', Social Science and Medicine, Vol. 6, No. 9, pp. 931-940.

Radio Cooperativa. Plan "Hospital amigo" aumentará a seis las horas de visitas familiares a pacientes. Disponible en www.Cooperativa.cl. 2 enero 2007

SEN G. Empowerment as an appraoch to poverty. Ginebra: Organización 
Mundial de la Salud. (Documento de Trabajo), 1997.

STROMQUIST, N. La búsqueda del empoderamiento: en qué puede contribuir el campo de la educación. En Denman c., Araneda, P., y Cornejo, E. Región y sociedad Vol. XI (18): 192, 1999.

TABORDA M, Copertari S, Ruiz Briz E, Gurevich E, Firpo V. Ciencias sociales e interdisciplinariedad: relación entre teoría y práctica, 2005. Disponible en: http://www.puentes.gov.ar/educar/servlet/Downloads/s_colecciones fin/aun0402.pdf (Consulta: 23 de mayo de 2007).

UNIVERSIA R/R Benito Baranda critica el actual sistema social y sus políticas. Disponible en www.universia.cl. (Consulta: 15 de octubre 2009)

Unidad de participación social de la Subsecretaría de redes asistenciales del MINSAL

VAN LEEUWEN, T. Three models of interdisciplinarity. In WODAK, R.; CHILTON, P. (Orgs.), 2005, p. 3-18.

WALLERSTAIN, N. What is the evidence on Effectiveness of Empowerment to improve health? (HEN), Copenhagen: WHO Regional office for Europe. 2006

WALLERSTAIN, N. Empowerment to reduce health disparities. Scandinavian Journal of Public health, 59:72-7, 2002

WALLERSTEIN, N. Powerlesss, Empowerment and Health: Implications for Health

Promotion Programs. American Journal of Health Promotion. Vol. 6. № 3, Ene-Feb

1992

WHITE, P. R. Beyond modality and hedging: A dialogic view of the language of intersubjective stance. Text, 23(2), 259-284. 2003 (Special issue).

\#, M. Percepción de la población pobre de Santiago sobre la atención de salud en Chile al año 2008 y visión evolutiva desde el año 2003. Disponible en www.universia.cl/portada/.../noticia actualidad - Consulta: 9 abril 2009. 